\title{
A parent-led intervention to reduce anxiety in autistic children with a severe to profound intellectual disability: protocol for the LADDERS pilot feasibility trial
}

\section{Jane Waite}

Aston University https://orcid.org/0000-0002-8676-3070

Joanne Tarver

Aston University

Effie Pearson ( $\nabla$ e.pearson1@aston.ac.uk)

Aston University https://orcid.org/0000-0002-4328-1653

Jessica Hughes

Aston University

Georgina Edwards

Aston University

Megan Bird

Aston University

Courtney Greenhill

Aston University

\section{Research Article}

Keywords: Anxiety, autism, intervention study, parent group, intellectual disability

Posted Date: July 27th, 2022

DOI: https://doi.org/10.21203/rs.3.rs-1258294/v2

License: (c) (i) This work is licensed under a Creative Commons Attribution 4.0 International License.

Read Full License 


\section{Abstract \\ Background}

There is a need for evidence-based approaches to reduce anxiety experienced by autistic children with severe to profound intellectual disability (ID). Avoidance of anxiety triggers, as a response to pronounced anxiety, occurs irrespective of age, background and neurodiversity. When avoidance is unhelpful, evidence-based anxiety reduction approaches aim to reduce it gradually (graded exposure), subsequently reducing anxiety. Combining graded exposure with emotional regulation techniques may be effective and acceptable for autistic children with severe to profound ID, if sensitive to needs and characteristics of autistic children. We have developed a 16-week, parent-led intervention (LADDERS) to reduce anxiety in this population of autistic children. LADDERS consists of psychoeducation, graded exposure-based tasks, and skills building, delivered utilising a person-centred approach. This study aims to assess whether LADDERS 1) reduces anxiety and 2 ) whether autistic children and parents find it acceptable and feasible.

\section{Method}

A single-site, multiple baseline, single case experimental study will be conducted. Participants will be parents of autistic children aged between 4-15 years. A minimum of 8 participants will be recruited through a research participant database, the Autistica Discover Network and social media. Once eligibility is confirmed, participants will be assessed at baseline, during the intervention and at a 2-month follow-up (week 24). The primary outcome measure will be a daily diary that assesses child anxiety.

\section{Discussion}

The study will provide preliminary evidence of whether LADDERS reduces anxiety in autistic children with severe to profound ID. Qualitative data from parents and child engagement will provide data on acceptability and feasibility.

\section{Full Text}

There is a dearth of intervention studies for anxiety in autistic children with severe to profound intellectual disabilities (ID), despite anxiety being highly prevalent in this population (Vereenooghe et al., 2018). There have been few studies addressing mental health for children with severe to profound ID based on psychological principles, and studies that have been conducted have focused on depressive symptomatology and tic frequency, rather than anxiety (Vereenooghe et al., 2018). The absence of evidence-based interventions for autistic children and adults with severe to profound ID is a significant concern given that mental health difficulties are elevated in the autistic population. Further, anxiety interventions have been identified as the top priority for autistic people and their families by the James Lind Alliance Priority Setting Partnership. 
Ongoing work within our team has examined the correlates of anxiety in minimally verbal autistic people and people with severe to profound ID via systematic reviews and in-depth parent interviews. The work has identified that anxiety is associated with avoidance of situations, behaviours that challenge, and ritualistic and repetitive behaviours (Tarver et al., 2021; Edwards, Tarver, Shirazi, Oliver \& Waite, 2019). These findings concur with the typically developing literature, in which avoidance of anxiety provoking situations has been identified as a hallmark characteristic of anxiety. This can include avoiding situations entirely (e.g. school refusal) or engaging in activities that lead to escape from situations (e.g. shouting in class) (Craske, Hermans, \& Vansteenwegen, 2006; Egger, Costello, \& Angold, 2003). Children experiencing anxiety also engage in safety behaviours to reduce anxiety, such as reassurance seeking, ritualistic behaviours or holding a special object (Craske et al., 2006; Kley, Tuschen-Caffier, \& Heinrichs, 2012).

Avoidance is an evolutionary response to threat and can be adaptive in some situations; however, avoidance can become excessive and maladaptive. Arnaudova, Kindt, Fanselow, and Beckers (2017) provide the following definition of avoidance as it presents in anxiety disorders: "execution of repetitive avoidance, which a) limits daily activities and impairs general functioning; $b$ ) can be provoked by stimuli, which do not pose any objective threat to the individual's health or well-being; $c$ ) is associated with a high level of distress." Excessive avoidance behaviour (task refusal, selective mutism) is often a trigger for parents and carers seeking support for their children, because of the impact behaviours associated with avoidance have on life activities for the child, parents and educators (Dymond \& Roche, 2009). Avoidance also reduces the likelihood of a child developing coping skills to manage avoided situations and it perpetuates the cycle of anxiety (Hayes \& Wilson, 1994). Even subtle avoidance behaviours may impair future learning that a stimulus that has been previously seen as threatening is no longer threatening. Therefore, during anxiety treatment, parents and children are encouraged to gradually reduce avoidance behaviours while supported by a health professional (Piccirillo, Taylor Dryman, \& Heimberg, 2016). A carefully conducted anxiety intervention encourages gradual reduction of avoidance so that an individual does not experience overwhelming levels of distress during the intervention, in contrast to controversial flooding techniques (Otto \& Hofmann, 2010). The aim of an anxiety intervention is to reduce excessive anxiety that may affect the individual's health and wellbeing. The aim is not to expose individuals to pain experienced primarily in response to sensory sensitivities with a view to reducing these sensitivities (sensory desensitization, e.g. Koegel, Openden \& Koegel, 2004).

While there are no evidence-based approaches for the treatment of anxiety in autistic children with severe to profound ID, there are existing behavioural interventions for escape-maintained behaviours that challenge (Hanley, Iwata, \& McCord, 2003). Escape-maintained behaviours often occur in autistic children with severe to profound ID when they are presented with a non-preferred task or situation which poses a challenge to them (e.g. hospital appointments or aspects of the morning routine). Interventions for 
escape-maintained behaviours often involve reducing task demands to a level that the child can manage without engaging in escape-maintained behaviours or experiencing excessive anxiety or distress, while prompting the individual to complete the task. Overtime, the demands of the situation are gradually increased, known as 'demand fading' (e.g. gradually increasing the time spent in school), as the association between engaging in behaviours that challenge and the removal of the demand is weakened (Hagopian, Boelter, \& Jarmolowicz, 2011).

Interventions for escape-maintained behaviours are rarely framed as interventions that reduce avoidance or anxiety in the autism and ID literature; however, there is substantial overlap between the steps followed in these interventions and anxiety focused interventions used with typically developing children (incremental exposure to the feared stimulus without experiencing aversive consequences). It is possible that some autistic individuals with ID engage in escape behaviours because of underlying anxiety related to a stimulus. Therefore, for these individuals, traditional behavioural interventions for escape behaviours may be effective because the intervention gradually exposes them to avoided stimuli and reduces anxiety.

Acknowledgement that anxiety reduction may be occurring during these evidence-based behavioural interventions is important for several reasons: 1 ) parents, carers or educators perceptions of why behaviour occurs has been shown to impact how they respond (Hassall \& Rose, 2005); 2) acknowledgement of anxiety may modify therapist approaches to intervention, i.e. reducing safety behaviours or increasing coping skills; 3 ) therapeutic approaches that overlook anxiety may under emphasise the role of parents' emotional experiences on the development and maintenance of child anxiety. Parental reinforcement of avoidance strategies can contribute to the maintenance of child anxiety (Cobham, Dadds, \& Spence, 1998; Rapee, 1997; Siqueland, Kendall, \& Steinberg, 1996).

Study aim: We have developed a manualised, parent-led intervention package, based on evidence-based strategies, designed to reduce anxiety-related avoidance in autistic children with a severe to profound intellectual disability who speak few or no words. The 16-week intervention package has been developed alongside clinicians and parents and has been designed to be appropriate for use within NHS services by behavioural support teams and mental health workers. The intervention integrates evidence-based behavioural interventions for escape-maintained behaviours from the ID literature with existing anxiety interventions from the typically developing literature (e.g. graded exposure). The intervention also includes components addressing the role of parental anxiety and avoidance in the perpetuation of the anxiety cycle. In the current study, we plan to pilot test the intervention using a multiple baseline single case study design to assess the ability of the intervention to reduce anxiety and avoidance behaviours in autistic children and their caregivers. We will also be collecting data regarding the acceptability and 
feasibility of the intervention through parent interviews following their involvement in the intervention, and via our observations of child engagement during the intervention.

\section{Patient and public involvement:}

During the development stages, seventeen parents of autistic children who speak few or no words assisted with the design and content of the intervention through focus groups and interviews. Resulting feedback was considered and discussed by the research team and subsequent adaptations to the intervention manual were made.

\section{Trial design:}

A single-centre multiple baseline, single case experimental study will be conducted. The study will be delivered online and in the participant's home in the United Kingdom. The start of the intervention will be staggered across participants to establish if changes in anxiety related symptoms and reduction in avoidance behaviours are due to the intervention rather than other temporal factors. This will also allow for analysis of whether behaviour change occurs prior to direct intervention with the child or during parent-focused sessions. Multiple baseline designs are a robust and effective way of demonstrating the utility of interventions with a small subset of individuals (Gast, Lloyd, \& Ledford, 2018), and have been used extensively with children and adults with intellectual disabilities (Gunning \& Espie, 2003; Hua et al., 2018; Ingersoll, Walton, Carlsen, \& Hamlin, 2013; Kim \& Kwon, 2018; Mevissen, Didden, Korzilius, \& de Jongh, 2017; Moskowitz et al., 2017; O'Handley, Ford, Radley, Helbig, \& Wimberly, 2016)

\section{Participants}

Participants will be a minimum of eight (maximum of 20; depending on attrition across the study) parents of autistic children with moderate to profound ID, the autistic child and their teacher. Participants will be recruited via a participant database held at Aston University, the Discover Network held by Autistica and through adverts on social media platforms held by the research team and by associated charities.

\section{Participant identification and recruitment process}

Initially, participant databases held by the PI and the research team at Aston University will be screened for participants meeting the following screening criteria: 
Screening criteria:

- Parents/carer of child aged between 4-15 years

- Child has clinical diagnosis of autism according to parent report or will meet cut-off for autism spectrum disorder on the Social Communication Questionnaire (data available from our current Aston database or collected during the initial screening of participants not previously known to the research team).

- Child speaks few words or odd words only on Wessex questionnaire (as an initial proxy measure of intellectual disability).

All potential participants who meet these criteria will be sent an invitation letter and an expression of interest form. Participants recruited through social media and the Autistica Discover Network will be encouraged to contact the research team using contact details provided.

For all families who have expressed an interest, a member of the research team will telephone participants to further assess study eligibility based on the following criteria which will be assessed via an eligibility interview schedule:

\section{Inclusion criteria}

1. Parent/carers of children 4-15 years with a clinical diagnosis of autism or meeting cut-off for autism spectrum disorder on the Social Communication Questionnaire

2. Parent/carers of children who speak few words or odd words only on the Wessex Questionnaire (this is for initial screening purposes. The presence of intellectual disability will be assessed during the baseline assessment stage of the intervention)

3. Parent reports that child experiences anxiety on 'more days than not' or reports that anxiety significantly impacts the child's quality of life on a weekly basis

4. Parents report that their child engages in avoidance or escape behaviour, in response to situations that appear to be related to anxiety (to be further assessed on entry into the study).

5. Parent agrees to child's GP and psychology/behavioural/psychiatry team (if applicable) being advised of involvement in the study.

6. Child currently living with consenting parent/carer full-time 


\section{Exclusion criteria}

1. Child has started any medication within 6 weeks of baseline measurement for any psychiatric diagnosis or for behavioural management reasons or there are planned medication changes for the duration of the intervention period

2. Child is in receipt of current focused regular input (fortnightly or more) from a clinical psychologist or related team specifically for anxiety, or that such input is planned to start during the time that the child is enrolled in the intervention

3. Current social services involvement with family for the purposes of child protection (active safeguarding concerns)

4. Parent/carers who do not have sufficient literacy to understand study questionnaires or engage in research related activities

5. Reliable improvements in anxiety during baseline period (i.e., a negative trend during the baseline period that concurs with reliable change on a questionnaire measure of anxiety).

A consensus meeting between the research team will be held to decide on the final decision of eligibility based on the criteria described above and information provided in the eligibility interview. Families who are deemed eligible will be invited to then provide consent.

\section{Intervention}

\section{LADDERS}

The LADDERS intervention is a 16-week parent-led programme which aims to reduce anxiety and anxiety avoidance behaviours in autistic children with a severe to profound intellectual disability who speak few or no words. It has been developed using literature on interventions for anxiety and anxiety avoidance in typical development and theories of escape-avoidance behaviours in intellectual disability. It has been further refined and developed based on feedback from families and clinicians. Importantly, the intervention is sensitive to the needs of the autistic population, focusing on anxiety that is excessive and does not pose any objective threat to the individual's health or well-being.

The intervention package comprises four key stages: 1) a psychoeducation component with the parent (weeks 1-5); 2) a formulation stage (week 6-9);3) the implementation of strategies (weeks 10-16) and 4) feedback and review of the intervention (weeks 16). The psychoeducation component (stage 1) focuses on educating parents about anxiety including identifying anxiety, maintenance of anxiety and influence of the parent's behaviour on child's anxiety. These sessions will encourage parents to apply the principles learned to their own situation. Stage 2 will focus on the formulation and development of strategies that 
parents will be using with their child in the following weeks. This will include the development of a graded exposure hierarchy ladder as well as having a discussion with the parents about any possible anticipated barriers to completing the exposure-based tasks. In stage 3, parents will work through the graded exposure ladder with their child with check-ins and support from the psychologist. In stage 4, the psychologist will review the intervention with the parent and discuss the next steps, signposting to further information and support. The intervention is delivered via 60-minute, weekly sessions in a community setting. Further information about the LADDERS intervention will be published in a separate manuscript.

\section{Procedure}

The full outline of the procedure participants will follow is outlined in figure 1. After the initial contact and pre-screening of eligible participants as described above, research staff will obtain informed consent from parents (and assent from the person they care for, wherever possible) and conduct baseline assessments to confirm eligibility. During baseline assessment, anxiety related behaviours will be monitored daily for either 6,10 or 14 days via questions from validated measures of anxiety and behaviour (described below). In this nonconcurrent multiple baseline design, the first participant will be randomised to one of the three baseline lengths, and when the next participant becomes available, they will be randomised to one of the remaining lengths (e.g. Watson \& Workman, 1981). An online research randomiser will be used to randomise participants (Urbaniak, Plous \& Lestik, 2007). The randomisation process will begin again once all baseline lengths have been used. If the research team are unable to make contact with parents for a period of longer than 5 days during the baseline period, or parents do not submit their daily scores for a period of 5 days or more, they will be advised they are unable to proceed into the intervention.

Parents will also be asked to complete the Anxiety Depression and Mood Scale (ADAMS; Esbensen et al., 2003) at the start and end of their baseline period. The generalised anxiety and social avoidance subscales of this measure will be used to detect reliable change in anxiety levels over the baseline period and guidance from Evans, Margison and Barkham (1998) will be used to deem if change is significant or not.

To proceed into the intervention, the baseline data collected from parents will need to indicate a clear trend during the baseline period (e.g., stable, variable flat, worsening, or improving). If a trend cannot be established in the initial baseline period, the baseline period will be extended to up to 21 days until the pattern is clear. The direction and extent of the trend will determine whether or not the participants can proceed to the intervention (further information about how this will be assessed and the criteria can be requested from the corresponding author). 
For those who proceed to the intervention from the baseline, pre-intervention measures will be completed (see table 1) and a conversation will be had with the family about which mode of delivery of the intervention would be most appropriate. In response to the COVID-19 pandemic, the intervention sessions will be delivered in two ways; 1 ) complete online delivery via video-conferencing software or 2) a hybrid delivery where intervention sessions are conducted online via video-conferencing software but some components such as child observations and direct assessments will be conducted at the participant's home. Once the mode is selected, participants will take part in the 16-week intervention programme. Postintervention questionnaires and feasibility and acceptability interviews will be conducted in week 16 and at a 2-month follow-up (week 24). An additional observation of child anxiety in Week 24 will be conducted as a further measurement of anxiety following intervention.

For all measures in the study, parents will have the option to complete them either online using a secure survey programme (Qualtrics) or via a hard-copy questionnaire pack.

\section{Outcomes}

Table 1 displays measures that are being used in the study and when they will be completed.

\section{Primary research outcome}

The primary outcome measure is parent report of daily child anxiety completed daily via an anxiety daily diary. This anxiety daily diary has been developed based on questions from the Subjective Units of Distress (Wolpe \& Lazarus, 1966) and the Escape Behaviour Questionnaire (EBQ; based on Hyman, Oliver \& Hall, 2000). Parents will complete up to seven questions (depending on yes/no answers), with the first two asking them to rate their child's anxiety from 0-100 for that day using an adapted, operationalised, scale from Back et al. (2014). The remaining questions concern the presence of any related behaviours (e.g. engagement in behaviours that challenge and/or avoidance behaviours) and ask follow-up Likertscale questions regarding frequency and context. The daily diary will be completed by the parent from week 1 and will continue for two weeks (week 18) after the last intervention session in week 16. This is so that any delayed or carryover effects from the intervention can be assessed. From week 8 , parents will be asked to complete five additional questions as part of the diary. These are related to the work they will be doing using the ladder detailing the hierarchy exposure-based tasks and focus on stage of the ladder, severity of anxiety at that stage, duration of task, strategies used and parental confidence. 


\section{Secondary research outcomes}

\section{Observed child anxiety}

Five-minute observations of the child in a situation or context that the parent has reported can elicit mild anxiety in the child, and which is related to the identified target situation or context will be conducted during weeks 2, 9, 14 and 24. With parental consent, observations will be video recorded to allow for later analysis and coding of the observations. These observations will be used to validate parental report. For each participant, an individualised checklist of anxiety markers will be created using pre-existing measures of anxiety (e.g. Briggs-Gowan et al., 2011; Richards et al., 2009). The checklist will be used by a coder to assess the presence of each behaviour for that child during the recorded observation. All coding of the observations will be done after all data collection. The coder will be independent of the research project and blind to the week when the observation was conducted, to reduce potential bias.

\section{Parent-reported child emotional and behavioural characteristics}

During weeks 1, 16 and 24 parents will be asked to complete a number of measures relating to a variety of emotional and behavioural characteristics of their child. Changes in these characteristics are associated with anxiety so monitoring the nature of any changes and the direction of these changes will provide additional information for interpreting each participant's response to the intervention. Their child's mental health symptomology will be measured using the Mood Interest and Pleasure Questionnaire (MIPQ; Ross \& Oliver, 2003), the ADAMS (Esbensen et al., 2003) and the Clinical Anxiety Screen for Intellectual Disability (CIASP-ID; Waite et al., in development). Child sensory processing will be measured by the Sensory Experience Questionnaire (SEQ; Baranek et al., 2006). The scores on this questionnaire will also be used to assess whether avoidance is due to anxiety or direct exposure to a sensory aversive stimulus (due to the stimuli being painful/physically uncomfortable to an autistic child). Child repetitive behaviour will be measured by the Repetitive Behavior Questionnaire - 2 (RBQ-2; Leekam et al., 2007). Child behaviours that challenge will be measured by the Challenging Behaviour Questionnaire (CBQ; Oliver et al., 2007). Child sleep behaviour will be measured by the Children's Sleep Habits Questionnaire (CSHQ; Owens et al., 2000). All these measures are appropriate and developed for individuals with severeprofound intellectual disability. 
Table 1. Measures completed during the feasibility study including stage of completion.

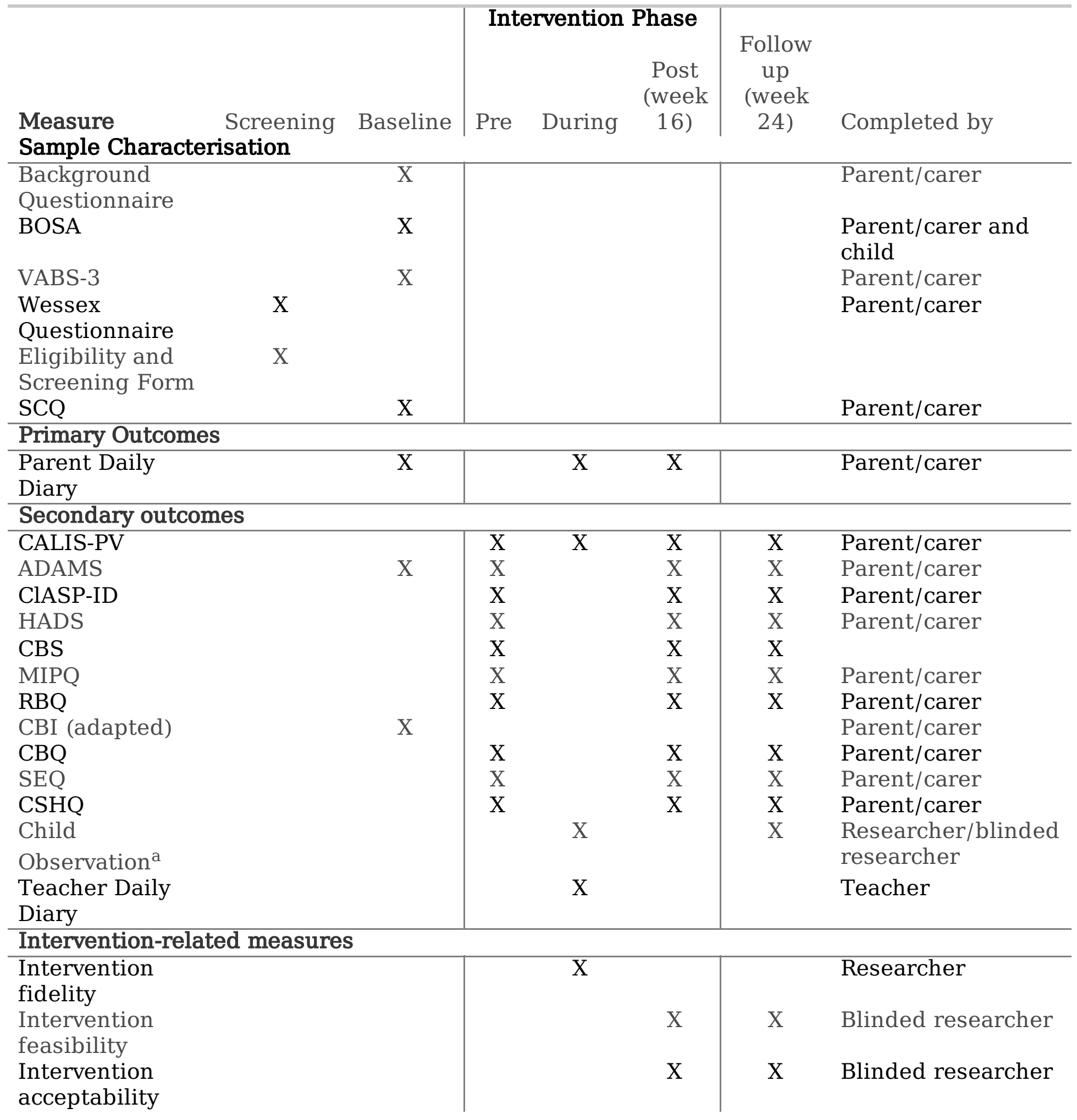

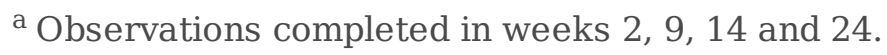

Abbreviations: BOSA, Brief Observation of Symptoms of Autism; VABS-3, Vineland Adaptive Behaviour Scales, $3^{\text {rd }}$ Edition; SCQ, Social Communication Questionnaire; CALIS-PV, Child Anxiety Life Interference Scale Preschool Version; ADAMS, Anxiety Depression and Mood Scale; ClASP-ID, Clinical Anxiety Screen for Intellectual Disability; HADS, Hospital Anxiety and Depression Scale; CBS, Controllability Beliefs Scale; MIPQ, Mood Interest and Pleasure Questionnaire; RBQ, Repetitive Behaviour Questionnaire CBI, Challenging 
Behaviour Interview CBQ, Challenging Behaviour Questionnaire SEQ, Sensory Experience Questionnaire CSHQ, Children's Sleep Habits Questionnaire.

Parent-reported parent wellbeing and family functioning

During weeks 1, 16 and 24 parents will be asked to complete measures that ask about their own wellbeing (Hospital Anxiety and Depression Scale (HADS); Zigmond \& Snaith, 1983) and their perceptions of escape-maintained behaviours that challenge (Controllability Beliefs Scale (CBS); Dagnan et al., 2004). Parents will also be asked to report on the impact of anxiety on family functioning. This will be measured by the Child Anxiety Life Interference Scale (CALIS-PV; Gilbertson et al., 2017) and will be completed weekly during the intervention period (weeks 1-16).

\section{Teacher-reported child anxiety}

Teachers of children who are participating in the intervention will be asked to complete a daily diary for the duration of the intervention period (weeks 1-16). The questions will ask teachers to provide an estimate of how much anxiety the child they teach has experienced on that day on a scale from 1-100. This is the same scale that is used in the parent daily diaries. These diaries will be used to assess generalisability across settings and potentially validate parental report (i.e. parents and teachers are in agreement about reduction of anxiety).

\section{Parent reports on feasibility and acceptability}

Brief semi-structured telephone interviews will be conducted with parents following completion of postintervention outcome measurement (week 16) and long-term outcome measurement (week 24). The interviews will be conducted by a member of the wider research team who is independent from the intervention project (will not be involved in the intervention design, delivery or collection of outcome measurement). The interview schedule explores parental feelings about the acceptability of the intervention, their views on the content and length of study assessments, and their continued use of the strategies that they learnt as part of the intervention.

\section{Sample characterisation measures}

Demographic information about the family is obtained at baseline. The child's level of adaptive ability (a proxy of intellectual disability) will be measured using the Vineland Adaptive Behaviour Scales - Third Edition (VABS-3; Sparrow et al., 2016). An adapted version of the Challenging Behaviour Interview (CBl; Oliver et al., 2013) will be conducted to characterise escape-avoidance behaviours in the sample, and to identify any that may be driven by anxiety. The CBI identifies the presence of behaviours that challenge 
and the duration, frequency and severity of these behaviours. It is validated for individuals with severe to profound ID (Oliver et al., 2003). The original version has been adapted for the pilot study and includes an additional question which asks if the behaviour that challenges is in response to anxiety. Three questions from the Questions about Behavioural Function questionnaire (Paclawskyjet al., 2000) are also included in the adapted $\mathrm{CBI}$ to ascertain if the function of the identified behaviours that challenge served an escape/avoidance function. Autism characteristics will be assessed through the Social Communication Questionnaire (SCQ; Rutter et al., 2003) and the Brief Observation of Symptoms of Autism (BOSA; Lord et al., 2020).

Although the Autism Diagnostic Observation Schedule-2 (ADOS-2; Lord et al., 2012) is the gold standard observation for assessing autism, due to the uncertainty of the COVID-19 pandemic, the current study will use the BOSA instead of the ADOS-2. This is to ensure consistency across participants and as a contingency to any changes in pandemic-related restrictions (note: the ADOS-2 cannot be administered using facemasks or coverings). The BOSA was developed by Dr Catherine Lord (co-author of the ADOS-2) and her team. It is adapted from the ADOS-2 and another autism assessment the Brief Observation of Social Communication Change (BOSCC; Grzadzinski et al., 2016). It is delivered by parents who are instructed by researchers or clinicians who can maintain a social distance.

\section{Intervention adherence}

Intervention component checklists will be created for each treatment session at the end of Stage 1 of this study. An independent observer will complete the intervention checklist for a randomly selected $20 \%$ of intervention sessions to ensure that treatment is being delivered as per protocol.

The researcher delivering the intervention sessions $(\mathrm{JH})$ will receive frequent clinical supervision from the study's PI (JW, clinical psychologist) to ensure adherence to the protocol and manual. If additional researchers deliver the intervention, they will also be provided with supervision to reduce variability in therapist effects.

\section{Planned analyses}

Following a bottom-up analysis of single-case experimental designs (SCED; (Parker \& Vannest, 2012) the results will be graphically represented. The scores obtained on assessments in the baseline and treatment phases will be compared using Tau-Ufor each participant (Parker, Vannest, Davis, \& Sauber, 2011). Tau- $U$ is a nonoverlap effect size that is suitable for non-parametric data. Tau- $U$ scores range from -1 to 1 and can be interpreted as the proportion of data that improved between two phases in a study (Parker et al., 2011). To obtain an overall estimate of the effect size of the intervention, the design- 
comparable effect size for multiple-baseline designs developed by Pustejovsky, Hedges, and Shadish (2014) will be computed. This standardised mean difference effect size for SCED shares the same metric as the Cohen's $d$ typically used in group designs. This $d$-statistic requires at least three cases for computation, and corrects for small sample bias using Hedges' g. Analyses will be completed using SPSS and R.

\section{Harm, adverse events and clinical concerns}

No serious adverse events are anticipated as a result of this intervention. Whilst temporary changes in child behaviour could be apparent, these should not be different to behaviours that most parents will have experience of managing on a daily basis. In between intervention sessions, if parents are planning to conduct graded exposure exercises, they will be encouraged to stay in touch with the research team via text or phone, particularly if they are experiencing difficulties or want advice. Parents will be encouraged to use the list of warning signs and list of calming strategies created as part of the intervention to help ensure that they are always working in their relative comfort zone. Parents will be advised to not address situations that will cause their child a high level of distress.

Parents will be asked at each session if there are any particular concerns or significant adverse changes in their child's behaviour since the beginning of the graded exposure tasks. If concerns or significant changes are reported by the parent, a case report form will be recorded in the site file and the study PI (JW, clinical psychologist) notified, with appropriate action taken. All staff delivering the programme are employed by the sponsor and will follow the Standardised Operating Procedures outlined by the sponsor (Aston University). These procedures are compliant with Good Clinical Practice (GCP) and Health Research Authority (HRA) guidelines for safety reporting. Child behaviour and parental well-being outcome measures will serve as measures of adverse events.

\section{Data management, confidentiality and access}

All participants will be given a unique ID number for the study. All digital data resulting from the study (e.g. video- and audio-recordings of intervention sessions, online questionnaire data) will be stored on a secure online drive hosted by the sponsor (Aston University). Only members of the research team will have access to the online drive. Any paper records will be filed pseudo-anonymously by the participant's study ID number and stored in secure locked cabinets in the study PI's office at Aston University. The data from paper records will be entered on statistical analysis software that will also be stored on the secure online drive. Consent forms will be stored separately from all study data. Personal details (eg, name, address and telephone numbers) will be stored on the online secure drive in a separate passwordprotected file and linked by the unique participant ID number. 
Video and audio recordings arising from this study will be downloaded from cameras and Dictaphones and stored on a secure, encrypted Aston University laptop at the end of the intervention session. If data is requiring transporting (i.e. from a home visit), the laptop will be returned to the University within 48 hours (unless it is a weekend). Virtual intervention sessions will be conducted using sponsor approved, secure video-conferencing software (Microsoft Teams) and using an encrypted Aston University laptop. All recorded sessions will immediately be uploaded to the secure, online drive and filed by the participant's unique study ID number.

Staff who are delivering this intervention, including the PI, therapist and research staff involved in screening, eligibility, baseline and the intervention, will have access to the project folders and data collected during this study. Clinical information will not be shared without written consent from the participant, unless there is significant concern as to the safety and welfare of a participant or their child (e.g. a safeguarding concern), expect as necessary for auditing by the Sponsor (Aston University), its designee or regulatory authorities.

\section{Dissemination plan}

Findings from the study will be written up for publication in peer-reviewed journals and translated into lay summaries to be distributed via the FIND website (www.findresources.co.uk) and Autistica. Additionally, findings will be presented at national and international conferences.

\section{Discussion}

There is an absence of evidence-based, manualised interventions to reduce anxiety and avoidance in autistic children with a severe to profound intellectual disability who speak few or no words. To address this gap, the LADDERS intervention programme aims to provide an evidence-based approach by providing parents with a foundation of knowledge behind anxiety maintenance and the role of parental anxiety, as well as utilising graded exposure strategies to reduce anxiety and avoidance related behaviours in their autistic child. LADDERS has the potential to improve psychological outcomes for autistic children with severe to profound intellectual disabilities and their families by providing parents with long-term strategies and skills that can be applied in future scenarios and a range of contexts. The present study will provide data on the effectiveness, acceptability and feasibility of the LADDERS intervention package to inform the design and sample size for a full-scale trial.

\section{Trial Status}

The first participant consented to the LADDERS trial on 11th November 2021 and is anticipated to finish participation on $18^{\text {th }}$ July 2022 (participant end date information added 01/05/2022). Recruitment has been extended until $31^{\text {st }}$ May 2022 (original end recruitment date was $31^{\text {st }}$ March 2022. Extension date added 01/05/2022). 


\section{References}

Arnaudova, l., Kindt, M., Fanselow, M., \& Beckers, T. (2017). Pathways towards the proliferation of avoidance in anxiety and implications for treatment. Behaviour Research and Therapy, 96, 3-13. doi:https://doi.org/10.1016/j.brat.2017.04.004

Back, S. E. (2014). Concurrent treatment of PTSD and substance use disorders using prolonged exposure (COPE): Therapist guide. Treatments That Work.

Baranek, G. T., David, F. J., Poe, M. D., Stone, W. L., \& Watson, L. R. (2006). Sensory Experiences Questionnaire: discriminating sensory features in young children with autism, developmental delays, and typical development. Journal of Child Psychology and Psychiatry, 47(6), 591-601.

Briggs-Gowan, M., Mian, N., Carter, A., \& Wakschlag, L. (2011). The Anxiety Dimensional Observation Schedule (Anx-DOS), Version 1.0. Northwestern University.

Cobham, V. E., Dadds, M. R., \& Spence, S. H. (1998). The role of parental anxiety in the treatment of childhood anxiety. Journal of consulting and clinical psychology, 66(6), 893.

Craske, M. G., Hermans, D. E., \& Vansteenwegen, D. E. (2006). Fear and learning: From basic processes to clinical implications: American Psychological Association.

Dagnan, D., Grant, F., \& McDonnell, A. (2004). Understanding challenging behaviour in older people; the development of the controllability Beliefs Scale. Behavioural and Cognitive Psychotherapy, 32(4), 501506.

Dow, D., Holbrook, A., Toolan, C., McDonald, N., Sterrett, K., Rosen, N., ... \& Lord, C. (2021). The Brief Observation of Symptoms of Autism (BOSA): Development of a New Adapted Assessment Measure for Remote Telehealth Administration through COVID-19 and Beyond. medRxiv.

Dymond, S., \& Roche, B. (2009). A contemporary behavior analysis of anxiety and avoidance. The Behavior Analyst, 32(1), 7-27.

Edwards, G., Tarver, J., Shirazi, A., Oliver, C., \& Waite, J. (2019). Behavioural profile of anxiety in autistic individuals with few or no words and individuals with genetic syndromes associated with intellectual disability. Paper presented at the International Society for Autism Research, Montreal, Canada.

Egger, H. L., Costello, J. E., \& Angold, A. (2003). School refusal and psychiatric disorders: A community study. Journal of the American Academy of Child \& Adolescent Psychiatry, 42(7), 797-807.

Esbensen, A. J., Rojahn, J., Aman, M. G., \& Ruedrich, S. (2003). Reliability and validity of an assessment instrument for anxiety, depression, and mood among individuals with mental retardation. Journal of autism and developmental disorders, 33(6), 617-629. 
Evans, C., Margison, F., \& Barkham, M. (1998). The contribution of reliable and clinically significant change methods to evidence-based mental health. Evidence Based Mental Health, 1(3), 70-72. doi:10.1136/ebmh.1.3.70

Gast, D. L., Lloyd, B. P., \& Ledford, J. R. (2018). Multiple baseline and multiple probe designs. In Single case research methodology (pp. 239-281): Routledge.

Gilbertson, T. J., Morgan, A. J., Rapee, R. M., Lyneham, H. J., \& Bayer, J. K. (2017). Psychometric properties of the Child Anxiety Life Interference Scale-Preschool Version. Journal of anxiety disorders, 52, 62-71.

Grzadzinski, R., Carr, T., Colombi, C., McGuire, K., Dufek, S., Pickles, A., \& Lord, C. (2016). Measuring changes in social communication behaviors: preliminary development of the Brief Observation of Social Communication Change (BOSCC). Journal of autism and developmental disorders, 46(7), 2464-2479.

Gunning, M., \& Espie, C. (2003). Psychological treatment of reported sleep disorder in adults with intellectual disability using a multiple baseline design. Journal of Intellectual Disability Research, 47(3), 191-202.

Hagopian, L. P., Boelter, E. W., \& Jarmolowicz, D. P. (2011). Reinforcement schedule thinning following functional communication training: Review and recommendations. Behavior Analysis in Practice, 4(1), 416.

Hanley, G. P., Iwata, B. A., \& McCord, B. E. (2003). Functional analysis of problem behavior: A review. Journal of Applied Behavior Analysis, 36(2), 147-185.

Hassall, R., \& Rose, J. (2005). Parental cognitions and adaptation to the demands of caring for a child with an intellectual disability: A review of the literature and implications for clinical interventions. Behavioural and Cognitive Psychotherapy, 33(1), 71-88.

Hua, Y., Yuan, C., Monroe, K., Hinzman, M. L., Alqahtani, S., Alwahbi, A. A., \& Kern, A. M. (2018). Effects of the Reread-Adapt and Answer-Comprehend and goal setting intervention on decoding and reading comprehension skills of young adults with intellectual disabilities. Dev Neurorehabil, 21(5), 279-289. doi:10.3109/17518423.2016.1139011

Hyman, P., Oliver, C., \& Hall, S. (2002). Self-injurious behavior, self-restraint, and compulsive behaviors in Cornelia de Lange syndrome. Am J Ment Retard, 107(2), 146-154. doi:10.1352/08958017(2002)107<0146:Sibsra>2.0.Co;2

Ingersoll, B., Walton, K., Carlsen, D., \& Hamlin, T. (2013). Social intervention for adolescents with autism and significant intellectual disability: initial efficacy of reciprocal imitation training. Am J Intellect Dev Disabil, 118(4), 247-261. doi:10.1352/1944-7558-188.4.247

Kim, J., \& Kwon, M. (2018). Effects of mindfulness-based intervention to improve task performance for children with intellectual disabilities. J App/ Res Intellect Disabil, 31(1), 87-97. doi:10.1111/jar.12333 
Kley, H., Tuschen-Caffier, B., \& Heinrichs, N. (2012). Safety behaviors, self-focused attention and negative thinking in children with social anxiety disorder, socially anxious and non-anxious children. Journal of behavior therapy and experimental psychiatry, 43(1), 548-555.

Koegel, R. L., Openden, D., \& Koegel, L. K. (2004). A systematic desensitization paradigm to treat hypersensitivity to auditory stimuli in children with autism in family contexts. Research and Practice for Persons with Severe Disabilities, 29(2), 122-134.

Kushlick, A., Blunden, R., \& Cox, G. (1973). A method of rating behaviour characteristies for use in large scale surveys of mental handicap. Psychological Medicine, 3(4), 466-478.

Leekam, S., Tandos, J., McConachie, H., Meins, E., Parkinson, K., Wright, C., .. . Couteur, A. L. (2007). Repetitive behaviours in typically developing 2-year-olds. Journal of Child Psychology and Psychiatry, 48(11), 1131-1138.

Lord, C., Rutter, M., DiLavore, P., Risi, S., Gotham, K., \& Bishop, S. (2012). Autism Diagnostic Observation Schedule: ADOS-2. Western Psychological Services.

Mevissen, L., Didden, R., Korzilius, H., \& de Jongh, A. (2017). Eye movement desensitisation and reprocessing therapy for posttraumatic stress disorder in a child and an adolescent with mild to borderline intellectual disability: A multiple baseline across subjects study. J App/ Res Intellect Disabil, 30 Supp/ 1, 34-41. doi:10.1111/jar.12335

Moskowitz, L. J., Walsh, C. E., Mulder, E., McLaughlin, D. M., Hajcak, G., Carr, E. G., \& Zarcone, J. R. (2017). Intervention for Anxiety and Problem Behavior in Children with Autism Spectrum Disorder and Intellectual Disability. J Autism Dev Disord, 47(12), 3930-3948. doi:10.1007/s10803-017-3070-z

O'Handley, R. D., Ford, W. B., Radley, K. C., Helbig, K. A., \& Wimberly, J. K. (2016). Social Skills Training for Adolescents With Intellectual Disabilities: A School-Based Evaluation. Behav Modif, 40(4), 541-567. doi:10.1177/0145445516629938

Oliver, C., McClintock, K., Hall, S., Smith, M., Dagnan, D., \& Stenfert-Kroese, B. (2003). Assessing the severity of challenging behaviour: psychometric properties of the challenging behaviour interview. Journal of Applied Research in Intellectual Disabilities, 16(1), 53-61.

Otto, M. W., \& Hofmann, S. G. (2010). Avoiding treatment failures in the anxiety disorders. New York, NY: Springer.

Owens, J. A., Spirito, A., McGuinn, M., \& Nobile, C. (2000). Sleep habits and sleep disturbance in elementary school-aged children. Journal of Developmental and Behavioral Pediatrics, 21(1), 27-36.

Paclawskyj, T. R., Matson, J. L., Rush, K. S., Smalls, Y., \& Vollmer, T. R. (2000). Questions about behavioral function (QABF):: A behavioral checklist for functional assessment of aberrant behavior. Research in developmental disabilities, 21(3), 223-229. 
Parker, R., \& Vannest, K. (2012). Bottom-up analysis of single-case research designs. Journal of Behavioral Education, 21(3), 254-265.

Parker, R., Vannest, K., Davis, J., \& Sauber, S. (2011). Combining Nonoverlap and Trend for Single-Case Research: Tau-U. Behavior Therapy, 42(2), 284-299. doi:https://doi.org/10.1016/j.beth.2010.08.006

Piccirillo, M. L., Taylor Dryman, M., \& Heimberg, R. G. (2016). Safety Behaviors in Adults With Social Anxiety: Review and Future Directions. Behavior Therapy, 47(5), 675-687.

doi:https://doi.org/10.1016/j.beth.2015.11.005

Pustejovsky, J. E., Hedges, L. V., \& Shadish, W. R. (2014). Design-comparable effect sizes in multiple baseline designs: A general modeling framework. Journal of Educational and Behavioral Statistics, 39(5), 368-393.

Rapee, R. M. (1997). Potential role of childrearing practices in the development of anxiety and depression. Clinical psychology review, 17(1), 47-67.

Richards, C., Moss, J., O’Farrell, L., Kaur, G., \& Oliver, C. (2009). Social anxiety in Cornelia de Lange syndrome. Journal of autism and developmental disorders, 39(8), 1155-1162.

Ross, E., \& Oliver, C. (2003). Preliminary analysis of the psychometric properties of the Mood, Interest \& Pleasure Questionnaire (MIPQ) for adults with severe and profound learning disabilities. British Journal of Clinical Psychology, 42(1), 81-93.

Rutter, M., Bailey, A., \& Lord, C. (2003). The social communication questionnaire: Manual: Western Psychological Services.

Siqueland, L., Kendall, P. C., \& Steinberg, L. (1996). Anxiety in children: Perceived family environments and observed family interaction. Journal of Clinical Child Psychology, 25(2), 225-237.

Sparrow, S. S., Balla, D. A., \& Cicchetti, D. V. (2016). Vineland III: Vineland adaptive behavior scales: American Guidance Service.

Urbaniak GC, Plous S, Lestik M (2007) Research randomiser. Available: http://

www.randomizer.org. Accessed 01 Nov 2021.

Vereenooghe, L., Flynn, S., Hastings, R. P., Adams, D., Chauhan, U., Cooper, S.-A., . . Jahoda, A. (2018). Interventions for mental health problems in children and adults with severe intellectual disabilities: a systematic review. BMJ open, 8(6), e021911.

Watson, P. J., \& Workman, E. A. (1981). The non-concurrent multiple baseline across-individuals design: An extension of the traditional multiple baseline design. Journal of Behavior Therapy and Experimental Psychiatry, 12(3), 257-259. 
Wolpe, J., \& Lazarus, A. (1966). Behavior therapy techniques: A guide to the treatment of neuroses. . New York:: Pergamon.

Zigmond, A. S., \& Snaith, R. P. (1983). The hospital anxiety and depression scale. Acta psychiatrica scandinavica, 67(6), 361-370.

\section{Declarations}

\section{Author contributions:}

$J W$ conceived of the study and is the grant holder. $J W, J T$, and $E P$ were involved in the study design and drafted the protocol for the study. $J W, J T, E P, J H, G E, M B$, and $C G$ contributed to the development of the intervention content. $J H, E P$ and $J W$ are involved in recruitment and eligibility screening. $J H$ is delivering the intervention. $J W$ is supervising the primary statistical analysis. EP drafted the manuscript and all authors read, made revisions and approved the final manuscript.

\section{Funding information:}

This study is funded by Autistica. This funding source had no role in the design of the study, the analyses, interpretation of the data, or decision to submit results. The funder will advertise the study for recruitment purposes but will have no further role in the selection or recruitment of participants.

\section{Competing interests:}

The study team wishes to declare a $\mathrm{COI}$ in that a positive outcome of the intervention may increase the likelihood of further funding for this work. This is an inherent COI in most feasibility/acceptability and pilot interventions that have not progressed yet to an RCT. Where possible, within the scope of the available funding and resources, the research team is acting to reduce any potential bias by ensuring transparency of data collection and analysis, and collecting validity data (e.g. observational data that will be coded by a researcher who is blind to the order that these observations were recorded). Week 24 qualitative evaluation data will not be collected or analysed by the therapist who delivered the intervention or the PI. No further COls to declare.

\section{Ethics approval:}

Obtained from Aston University Research Ethics Committee on 21/04/2020 (ref: UREC \#1597).

\section{Figures}


Families will be recruited through an existing participant database held at Aston University, Autistica's Discover Network and social media adverts. Eligible families will be sent a letter inviting them to take part in the study and an expression of interest form.

Parents/carers who express interest in the study will complete an eligibility assessment with a researcher to identify whether their child meets eligibility criteria. Screening measures will also be completed with parents/carers.

Completed screening and eligibility assessments will be taken to an eligibility consensus meeting with the research team, including the PI, to decide whether the intervention is suitable for the family.

If the child is eligible and their parent is still interested in taking part in the study, the parent will be provided with an information sheet and asked to complete a consent form.

Once eligibility and consent are established, the participant is randomly allocated to a baseline ( 6 , 10 or 14 days), and initial baseline measures are completed with the parent and their child.

The participant completes the baseline phase. If still eligible based on the trend of baseline data, the parent completes the pre-intervention measures.

The participant takes part in the 16-week intervention consisting of weekly 1-hour sessions. The parent also completes daily monitoring of their child's anxiety, behaviour and implementation and weekly monitoring of the impact of their child's anxiety

In week 16 the parent completes the post-intervention questionnaires and a feasibility and acceptability interview.

At a 2-month follow-up (week 24), the parent repeats the post-intervention questionnaires and completes a further feasibility and acceptability interview.

\section{Figure 1}

Participant identification, recruitment and procedure

\section{Supplementary Files}

This is a list of supplementary files associated with this preprint. Click to download.

- SPIRITChecklistLadders18.05.22.doc 